Arch. Tierz., Dummerstorf 42 (1999) 4, 353-363

Aus dem Hybridschweinezuchtverband Nord / Ost e.V. ' und dem Gut Vogelsang Dr.Gey \& Sohn GbR ?

UWE HÖHN ${ }^{1}$ und GOTTFRIED GEY ${ }^{2}$

\title{
Untersuchungen zum Einfluß der Geburtensynchronisation auf tiergesundheitliche Merkmale von Partussauen und Ferkeln
}

\begin{abstract}
Summary
Title of the paper: Studies on the influence of synchronization of parturition as with regard to features of the animal health of partus sows and piglets

The tests were carried out in a piglet production plant with 1275 sow places which is run on a 7-day-cycle based on the periodical farrowing system. The tests were splitted into 2 stages. In the first stage 263 controlled sows were farrowing spontaneously after a gestation period of $112.9 \pm 1.03$ days. 637 sows still awaiting birth did get preferably on the 114th day an injection initiating parturition of Cloprostenol in the dosages of $50 \mu \mathrm{g}(\mathrm{N}=219)$, $100 \mu \mathrm{g}(\mathrm{N}=206)$ resp. $175 \mu \mathrm{g}(\mathrm{N}=112)$.

Parturition did start on an average after $20.3 ; 24.4$ resp. 21.2 hours and the quota of farrowing sows up to 36 hours post injectionem did reach $95.4 ; 94.2$ resp. $97.2 \%$ (not significant). The partus sows treated biotechnically did show agalactica less often (less $8.8 \%)$ and did have a normal body inner temperature $(+8.7 \%)$. With an increasing partus period exceeding 4 hours the rate of stillbirth went up by $4 \%$ to $9.9 \%$. An equalization of litter was made. In the second stage 170 sows with spontaneous parturition did form the control group. 369 sows were given $50 \mu \mathrm{g}$ Cloprostenol on the 114th day of gestation and another 187 sows which did not farrow within 24 hours got another injection of $0.07 \mathrm{mg}$ Carbetocin in $1 \mathrm{ml}$ Depotocin. Amongst the sows treated with biotechnical initiation of parturition $8.2 \%$ did show less discharge. In both stages the rearing success of the sows treated biotechnically did exceed that one of the animals by 0.51 resp. 0.41 weaned piglets each litter $(p<0,05)$.
\end{abstract}

Key words: parturition, biotechnology, animal health, partus sows, piglets

\section{Zusammenfassung}

Die Untersuchungen erfolgten in einem Ferkelerzeugerbetrieb mit 1275 Sauenplätzen, welcher auf der Grundlage des periodenweisen Abferkelsystems im 7-Tage-Rhythmus bewirtschaftet wird. Sie gliederten sich in zwei Teile. Im ersten Abschnitt ferkelten 263 Kontrolltiere nach einer Trächtigkeitsdauer von 112,9 $\pm 1,03$ Tagen spontan ab. 637 Sauen mit noch ausstehender Geburt erhielten vorzugsweise am 114. Trächtigkeitstag eine geburtsauslösende Injektion von Cloprostenol in den Dosierungen $50 \mu \mathrm{g}(\mathrm{N}=219) ; 100 \mu \mathrm{g}(\mathrm{N}=206)$ resp. $175 \mu \mathrm{g}(\mathrm{N}=212)$. Die Geburtseintritte erfolgten im Mittel nach 20,$3 ; 24,4$ resp. 21,2 Stunden und die Anteile abferkelnder Sauen bis 36 Stunden post injektionem betrugen 95,$4 ; 94,2$ resp. $97,2 \%$ (nicht sign.).

Die biotechnisch behandelten Partussauen wiesen weniger häufig Milchmangel auf (minus $8,8 \%$ Vorkommen) und hatten zu einem um 8,7 höheren Prozentsatz eine normale Körperinnentemperatur. Mit steigender Geburtsdauer über 4 Stunden stieg die Totgeburtenrate von $4 \%$ bis auf $9,9 \%$ an. Es erfolgte ein Wurfausgleich. Im zweiten Abschnitt bildeten 170 Sauen mit Spontangeburten die Kontrollgruppe. 369 Sauen erhielten am 114. Trächtigkeitstag $50 \mu \mathrm{g}$ Cloprostenol, und weitere 187 Tiere, die nicht innerhalb von 24 Stunden ferkelten, eine weitere Injektion von $0,07 \mu \mathrm{g}$ Carbetocin in $1 \mathrm{ml}$ Depotocin. Von den Sauen mit biotechnischer Geburtsauslösung zeigten $8,2 \%$ weniger Ausfluß. In beiden Abschnitten übertraf das Aufzuchtergebnis der biotechnisch behandelten Sauen dasjenige der Kontrolltiere um 0,51 bzw. 0,41 Ferkel je abgesetzten Wurf ( $p<$ $0,05)$

Schlüsselwörter: Geburt, Biotechnik, Tiergesundheit, Partussau, Ferkel 
1. Einleitung

In der praktischen Schweinezucht bildet die Organisation des Abferkelgeschehens einen wichtigen Bestandteil von Bewirtschaftungsprogrammen, die die Tiergesundheit fördern. In einem Rückblick auf die Entwicklung der Schweineleistungskontrolle in Brandenburg würdigte RITTER (1998) das Wirken von F. HOFMANN, der schon 1935 ein zeitlich festgelegtes Deck- und Abferkelsystem forderte. Ziel war es, wegen der damals z.T. unzureichenden Ställe, Winterwürfe und damit Verluste zu vermeiden. Ende der 50er Jahre sollte er diese Gedanken in Jena zum periodischen Abferkelsystem weiterentwickeln (HOFMANN, 1959/60). Die hierauf basierende Gruppenabferkelung und Zyklogrammsteuerung der Ferkelerzeugung bietet eine Reihe von tierhygienischen, arbeitswirtschaftlichen und ergebniswirksamen Vorteilen. Sie fördert zugleich die rationelle Nutzung der zunehmend verbreiteten Schweinebesamung und die Bereitstellung größerer Ferkelpartien in marktgerechter Qualität. Angestrebt werden möglichst kurze Zeitspannen für die Abferkelungen der aufeinander folgenden Sauengruppen und eine kurze Geburtsdauer der Einzeltiere. Ersterem steht die natürliche Schwankungsbreite der Trächtigkeitsdauer entgegen (SCHLEGEL, 1978; HEIDLER und HÜHN, 1979). Mittels erprobter biotechnischer Verfahren läßt sich eine terminliche und quantitative Ausrichtung fortpflanzungsbiologischer Prozesse auf notwendige und wünschenswerte Produktionsabläufe unterstützen. Vielerorts hat seit den $80 \mathrm{er}$ Jahren die Gleichschaltung der Geburtseintritte bei den gruppenweise abferkelnden Sauen (Geburtensynchronisation) mittels sehr sicher wirkender Prostaglandin F $2 \alpha$ Präparate (PGF 2 $\alpha$ ) Eingang in das fortpflanzungsbiologische Herdenmanagement gefunden (HOLTZ, 1988; HÜHN und KÖNIG, 1989). Unter Großbestandsbedingungen erfuhren insbesondere die synthetischen PGF 2 $\alpha$-Analoga, die den Wirkstoff Clopostenol enthalten, eine umfassende Erprobung (HÜHN u.a. 1981; LUSKY u.a., 1987; PODANY u.a., 1987).

Ihre Injektion nahe dem physiologischen Geburtstermin gestattet einen weitgehenden Ausschluß unerwünscht langer Tragezeiten von über 116 Tagen. Mittels kombinierter Behandlungsprogramme aus PGF $2 \alpha$ plus geeigneten Oxytocin-Präparaten läßt sich eine weitere Komprimierung der Abferkeltermine und deren tageszeitliche Steuerung bewerkstelligen. Dabei wird die Wirkung von Oxytocin, welches den Sauen mit noch ausstehender Geburt im Abstand von 22 bis 26 Stunden nach Cloprostenol verabreicht wird (HÖRÜGEL u.a., 1988), durch diejenige eines Langzeitoxytocins (Depotocin ${ }^{\circledR}$, $1 \mathrm{ml}$ enthält $0,07 \mathrm{ml}$ des Wirkstoffes Carbetocin) noch übertroffen (BERNHARD u.a., 1993; SCHULZ, 1993). Unter Praxisbedingungen ostdeutscher Ferkelerzeugerbetriebe wird im Hinblick auf die Vitalität und Gewichtsentwicklung der Neugeborenen die Verfahrensvariante der partiellen Geburtsinduktion bevorzugt (ROOST u.a., 1986; SCHNURRBUSCH und HÜHN, 1994). Die geburtsauslösende Injektion erfolgt möglichst nicht vor dem 114. Trächtigkeitstag, so daß nur diejenigen Sauen biotechnisch behandelt werden, bei denen die pränatale Entwicklung und Ausreifung der Ferkel so weit abgeschlossen sind, daß keine Beeinträchtigung der Aufzuchtergebnisse befürchtet werden muß.

Unter diesen Umständen fallen bei partieller Anwendung der genannten Biotechnika 
sowohl spontane als auch medikamentös ausgelöste Geburten innerhalb der einzelnen Abferkelperioden der Sauengruppen an. Bislang fehlten vergleichende Untersuchungen darüber, ob bei den einbezogenen Tieren mit behandlungsbedingten Unterschieden in gesundheitlicher Hinsicht zu rechnen ist. Die nachfolgende Feldstudie verfolgte daher das Ziel, einen Beitrag zur Beantwortung dieser Frage zu leisten. Zugleich sollte unter dem pharmakologischen Aspekt einer noch effektiven Niedrigdosierung geprüft werden, ob die praxisübliche Cloprostenolmenge von $175 \mu \mathrm{g}$ je Partussau gesenkt werden kann.

2. Material und Methoden

Die Untersuchungen erfolgten in einer in Mecklenburg gelegenen Schweinezuchtanlage, welche dem Typenprojekt der in der Investitionsperiode der 70er Jahre errichteten 1275er SZA (Sauenzuchtanlagen mit 1275 Plätzen) entspricht (SCHEWE, 1998). Die Bewirtschaftung geschieht auf der Grundlage eines Produktionszyklogrammes im 7 Tage-Rhythmus und es fallen planmäßig 46 Abferkelungen in jeder Woche an. Die Anwendung langjährig erprobter Verfahren zur Zyklussteuerung hat zur Folge, daß sich die Erstbelegungen der Alt- und Jungsauen bei umfassendem Einsatz der künstlichen Besamung auf den Wochenanfang (Montag, Dienstag) konzentrieren, so daß der 114. Trächtigkeitstag (Tr.T.) im Mittel stets auf Mittwoch fällt. Bei den Sauen handelte es sich um Kreuzungstiere mit 50 \%igem Landrasse-Anteil. Die zutretenden Jungsauen entstammten der betrieblichen Aufzucht.

Die bis zum Mittwoch morgens anfallenden spontanen Abferkelungen wurden stets abgewartet.

Die Sauen mit noch ausstehenden Geburten erhielten eine Injektion von Cloprostenol zur Partusinduktion. Im ersten Teil der Untersuchungen wurden die drei Dosierungen $175 \mu \mathrm{g}$ (bisheriger Standard), $100 \mu \mathrm{g}$ bzw. $50 \mu \mathrm{g}$ Cloprostenol miteinander verglichen. Die Sauen mit einer längeren Reaktionszeit als 24 Stunden post injektionem erhielten am Donnerstag morgens eine Injektion von $1 \mathrm{ml}$ Depotocin.

Bei allen Geburten wurden die Geburtseintritte sowie die Zeit von der Austreibung des ersten bis zum letzten Ferkel (=Geburtsdauer) registriert. Bei der Berechnung der Trächtigkeitsdauer zählten der Tag der letzten Insemination als erster und der Geburtseintritt als letzter Trächtigkeitstag. Von jedem Wurf wurde die Anzahl insgesamt sowie lebend geborener Ferkel erfaßt. Daraus wurde der Anteil tot geborener Ferkel je Wurf errechnet. Nach fünfwöchiger Säugezeit wurde die Anzahl der abgesetzten Ferkel erfaßt. Nach Abschluß jeder Abferkelperiode wurde ein Wurfausgleich vorgenommen. Bei den Würfen wurde der Anteil notwendiger Behandlungen erfaßt. Bezüglich der Ferkelgesundheit ergaben sich Würfe ohne Behandlung, solche mit Behandlung einzelner Ferkel sowie solche, bei denen die Mehrzahl der Ferkel behandelt werden mußte.

Bei den Partussauen erfolgten systematische Messungen der Körperinnentemperatur an den ersten drei Tagen post partum und deren Klassifizierung in drei Gruppen: Normal (bis $\left.39,3^{\circ} \mathrm{C}\right)$, leicht erhöht $\left(39,4-39,9^{\circ} \mathrm{C}\right.$ ) bzw. stark erhöht (über $\left.39,9^{\circ} \mathrm{C}\right)$. Im weiteren wurden die Sauen mit Milchmangel registriert und die Anteile von Tieren mit unter- 
schiedlichem Schweregrad von Ausfluß (ohne; leicht; schwer) ermittelt.

Im ersten Teil der Untersuchungen wurden 263 Spontangeburten sowie 637 Cloprostenol- induzierte Geburten ausgewertet. Der Anteil von Sauen mit Geburtseintritten innerhalb einer vorgegebenen Zeitspanne (z.B. 36 Stunden) wurde als Partusrate bezeichnet (im Beispiel: $\mathrm{PR}_{36}$ ).

Im zweiten Teil der Untersuchungen standen 170 Spontangeburten weiteren 369 mit Cloprostenol allein sowie 187 mittels einer kombinierten Behandlung aus Cloprostenol und Depotocin ausgelösten Geburten gegenüber. Da nach den vorausgegangenen Befunden große Würfe im Mittel zu kürzeren Tragezeiten führen (HEIDLER und HÜHN, 1979), war von vornherein bei den vorliegend biotechnisch induzierten Geburten ab 114./115. Trächtigkeitstag mit geringeren Wurfgrößen als bei den spontan abferkelnden Sauen zu rechnen.

Auftretende Mittelwertdifferenzen wurden mittels t-Test und Verteilungsunterschiede von Partussauen und Ferkeln auf Klassen mit den genannten tiergesundheitlichen Kriterien mittels Homogenitätsprüfung (Chi-Quadrat-Test) auf Signifikanz geprüft. Der Versuchsplanung wurde ein Signifikanzniveau von $\mathrm{p}<0,05$ zugrundegelegt.

3.

Ergebnisse

3.1 Vergleich der Tierleistungen nach spontanen Abferkelungen und Cloprostenol-induzierten Geburten

Die bis zum vorgesehenen Termin der Geburtsinduktion registrierten spontanen Abferkelungen wiesen eine Trächtigkeitsdauer von 112,9 $\pm 1,03$ Tagen $(\bar{x} \pm s)$ auf.

Die geburtsauslösende Injektion erfolgte bei den drei Versuchstiergruppen bei annähernd gleicher Sauenanzahl mit $50 \mu \mathrm{g}$ (I), $100 \mu \mathrm{g}$ (II) resp. $175 \mu \mathrm{g}$ Cloprostenol (III) am Tr.T 113,9 $\pm 0,31$. Tabelle 1 enthält die Gegenüberstellung der Meßwerte zum Eintritt und zur Dauer der Geburten sowie die erzielten Wurfleistungen. Die Geburtsdauer je insgesamt geborenes Ferkel wurde als Mittelwert errechnet.

Tabelle 1

Geburtseintritte, Geburtsdauer und Wurfleistungen von Sauen nach spontanen bzw. Cloprostenol-induzierten Geburten (Onset and duration of parturition and litter traits of sows following spontaneous resp. Cloprostenol induced farrowing)

\begin{tabular}{|c|c|c|c|c|}
\hline \multirow[t]{2}{*}{ Merkmal } & \multicolumn{3}{|c|}{ Art der Geburtsauslosung } & \multirow[t]{2}{*}{ spontan } \\
\hline & $50 \mu \mathrm{g}$ & $100 \mathrm{mg}$ & $175 \mu \mathrm{g}$ Cloprostenol & \\
\hline Tierzahl (St.) & 219 & 206 & 212 & 263 \\
\hline Geburtseintrittt (h p.inj.) & $20,3 \pm 10,5$ & $24,4 \pm 26,9$ & $21,2 \pm 9,3$ & - \\
\hline Geburtsdauer (min.) & $254 \pm 155$ & $247 \pm 123$ & $253 \pm 144$ & $243 \pm 142$ \\
\hline Geburtsdauer je insg.geb.F (min.) & 23,8 & 24,6 & 24,8 & 22,0 \\
\hline insges.geb.Ferkel/Wurf (St.) & $10,63 \pm 3,39$ & $10,04 \pm 3,29$ & $10.18 \pm 3,26$ & $11,05 \pm 2,97$ \\
\hline leb.geb.Ferkel/Wurf (St.) & $10,06 \pm 3,22$ & $9,49 \pm 3,09$ & $9,75 \pm 3,24$ & $10,38 \pm 2,86$ \\
\hline tot geb.Ferkel/Wurf (\%) & 5,4 & 5,5 & 4,4 & 6,1 \\
\hline abgesetzte Ferkel/Wurf (St.) & $8,82 \pm 2,66^{\circ}$ & $9,09 \pm 2,25^{n}$ & $8,80 \pm 2,63^{x}$ & $8,21 \pm 3,49^{b}$ \\
\hline
\end{tabular}

ungleiche Buchstaben innerhalb einer Zeile kennzeichnen sign. Unterschiede $(\mathrm{p}<0,05)$

Zwischen den Tieren der Kontrollgruppe mit spontanen Geburten einerseits sowie den Versuchsgruppen mit biotechnisch ausgelösten Abferkelungen andererseits bestanden 
bei den aufgeführten Parametern des Geburtsgeschehens und der Wurfgröße bei Geburt nur zufallsbedingte Unterschiede. Der Wurfausgleich erfolgte innerhalb der Würfe jeweils der gleichen Abferkelperiode ohne Berücksichtigung der Art der Geburtsauslösung. Danach erzielten die Sauen mit Cloprostenol-induzierten Abferkelungen eine signifikant höhere Anzahl abgesetzter Ferkel. Als Bezugsgröße diente die Anzahl abgesetzter Würfe. Die Überlegenheit gegenüber der Kontrollgruppe betrug 0,61 (I); 0,88 (II) resp. O,59 abgesetzte Ferkel je Wurf (III). Die reduzierten Dosisstufen von Cloprostenol zur Geburtsauslösung führten $\mathrm{zu}$ vergleichbaren Anteilen von abferkelnden Sauen innerhalb der praktisch interessierenden Zeitspannen bis 36 Stunden nach der Injektion. In Tabelle 2 wurden die erreichten Partusraten zusammengestellt. Die PR 36 betrug in allen Versuchsgruppen über $94 \%$.

Tabelle 2 Partusraten nach Geburtsauslösung mit Cloprostenol mit unterschiedlicher Dosis (Partus rate following
Cloprostenol injection in different dose)

\begin{tabular}{llcc}
\hline & & Cloprostenol - Dosis & $175 \mu \mathrm{g}$ \\
\hline Partusrate (\%) & $50 \mu \mathrm{g}$ & $100 \mu \mathrm{g}$ & 55,2 \\
24 Std. post injectionem & 60,3 & 53,8 & 89,6 \\
30 Std. post injectionem & 86,3 & 84,5 & 97,2 \\
\hline 36 Std. post injectionem & 95,4 & 94,2 & \\
\hline
\end{tabular}

Bei den Partussauen und Saugferkeln wurden die in Tabelle 3 aufgeführten Verhältnisse zur Charakterisierung der tiergesundheitlichen Situation nach unterschiedlicher Geburtsauslösung ermittelt. Sie beziehen sich auf die in Tabelle 1 enthaltene Anzahl von Sauen und Würfen.

\section{Tabelle 3}

Angaben zur Tiergesundheit von Sauen und deren Würfen nach unterschiedlicher Geburtsauslösung (Parameters of animal health in sows and their litters following different induction of parturition)

\begin{tabular}{|c|c|c|c|c|}
\hline \multirow{2}{*}{$\begin{array}{l}\text { Merkmal } \\
\text { nnnm }\end{array}$} & \multicolumn{3}{|c|}{ Art der Geburtsauslösung } & \multirow[t]{2}{*}{ spontan } \\
\hline & $50 \mu \mathrm{g}$ & $100 \mu \mathrm{g}$ & $175 \mu \mathrm{g}$ Cloprostenol & \\
\hline \multicolumn{5}{|l|}{ Anteile von Sauen (\%) } \\
\hline mit Milchmangel & 13,7 & 10,7 & 12,3 & 21,0 \\
\hline norm. Körperinnentemperatur & 46,6 & 40,8 & 46,2 & 35,2 \\
\hline leicht erhöhte Temperatur & 32,9 & 35,9 & 35,4 & 35,9 \\
\hline stark erhöhte Temperatur & 19,2 & 19,4 & 15,6 & 23,6 \\
\hline ohne Ausfluß & 73,0 & 66,5 & 69,3 & 65,2 \\
\hline mit leichtem Ausfluß & 20,6 & 17,5 & 20,7 & 19,8 \\
\hline mit schwerem Ausfluß & 6,9 & 13,6 & 7,5 & 10,5 \\
\hline \multicolumn{5}{|l|}{ Anteile von Würfen (\%) } \\
\hline ohne notwendige Ferkelbehandlung & $34,8^{*}$ & $28,6^{b}$ & $26,4^{b}$ & $24,7^{b}$ \\
\hline Behandlung einzelner Ferkel & $39,7^{*}$ & $48,1^{b}$ & $48,1^{b}$ & $51,9^{b}$ \\
\hline Behandlung der Mehrzahl Ferkel & 14,6 & 14,1 & $14, \&$ & 13,8 \\
\hline
\end{tabular}

Die Sauen mit Cloprostenol-induzierten Geburten wiesen geringere Häufigkeiten im Vorkommen von Milchmangel und stark erhöhter Körperinnentemperatur auf. Bei zusammengefaßter Betrachtung der 3 Dosierungsstufen fielen die Cloprostenol-behandelten Gruppen mit 12,2\% Milchmangel deutlich weniger auf als die spontan abgeferkelten Tiere mit $21 \%$ Milchmangel; die Differenz von $8,8 \%$ war signifikant. Eine 
normale Körperinnentemperatur wiesen 45,8 \% der Partussauen nach biotechnisch induzierten Geburten im Vergleich zu nur 37,1 \% der Tiere nach Spontangeburten auf. Auch diese Differenz von 8,7\% erwies sich als signifikant. Hingegen traten bezüglich auftretender Ausflußraten in Puerperium keine behandlungsbedingten Unterschiede in Erscheinung.

In den Würfen von Sauen nach Geburtsauslösung mit $50 \mu \mathrm{g}$ Cloprostenol wurden deutlich höhere Anteile von gesunden Ferkeln registriert, die keiner tierärztlichen Behandlung bedurften, als in allen anderen Gruppen. Dieser Effekt setzte sich jedoch in den Dosis-Varianten $100 \mu \mathrm{g}$ bzw. $175 \mu \mathrm{g}$ Cloprostenol nicht fort.

Die Geburtsdauer der Sauen schwankte von weniger als einer bis über 7 Stunden je Partus. Im folgenden wurde für insgesamt 629 Würfe des ersten Versuches eine Zuordnung der Totgeburtenrate zu den Sauen mit unterschiedlicher Geburtsdauer vorgenommen. In Tabelle 4 erfolgte die Zusammenstellung. Sauen mit der geringsten Geburtsdauer wiesen eine mittlere Totgeburtenrate von $4 \%$ auf. Mit zunehmender Dauer der Geburten nahmen die Anteile tot geborener Ferkel signifikant zu.

Tabelle 4

Geburtsdauer und Anteil tot geborener Ferkel (Duration of parturition and share of dead born piglets)

\begin{tabular}{ccc}
\hline Geburtsdauer (Std.) & Anzahl Würfe (St.) & Anteil tot geborener Ferkel (\%) \\
\hline bis 4 & 376 & $4,0^{\mathrm{a}}$ \\
$>4$ bis 6 & 161 & $5,8^{\mathrm{b}}$ \\
$>6$ & 92 & $9,9^{\circ}$ \\
\hline
\end{tabular}

ungleiche Buchstaben innerhalb der Spalte kennzeichnen sign. Unterschiede $(p<0,05)$ Geburtsauslösung

Es wurde die im ersten Versuchsabschnitt als wirksam erkannte Niedrigdosis von 50 $\mu \mathrm{g}$ Cloprostenol allein sowie kombiniert mit einer 24 Stunden darauffolgenden Injektion von $1 \mathrm{ml}$ Depotocin geprüft und mit den Ergebnissen der Kontrolle (Spontangeburten bis 114 Tr.T.) verglichen. Die geburtsauslösenden Injektionen von Cloprostenol

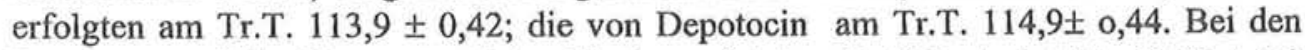
Reagenten auf die Cloprostenol-Applikation erfolgten die Geburtseintritte 13,98 \pm 7,2 Stunden post injectionem. Die Depotocin-Nachbehandlung hatte eine mittlere Reaktionszeit von 3,07 Stunden bis zum Beginn der Geburten zur Folge. Die Standardabweichung von $\pm 0,92$ Stunden drückt den Konzentrationsgrad der gleichgeschalteten Abferkelungen aus, welcher am Wochentag der Depotocin-Applikation (stets Freitags am Morgen) erreicht wurde. Die Meßwerte zur Geburtsdauer sowie die registrierten Wurfleistungen gehen aus Tabelle 5 hervor.

Die nach der Depotocin-Applikation registrierten Geburten waren im Durchschnitt kürzer als bei den Spontangeburten und Abferkelungen nach alleiniger CloprostenolGabe. Jedoch ergab die Relativierung auf die Wurfgröße keine unterschiedliche Geburtsdauer je insgesamt geborenes Ferkel. Die nach dem postpartalen Wurfausgleich erzielten Aufzuchtergebnisse der biotechnisch behandelten Partussauen erwiesen sich 
gemäß Zusammenfassung der Teilgruppen I ( $50 \mu \mathrm{g}$ Cloposterol allein) und II ( $50 \mu \mathrm{g}$ Cloposterol + $1 \mathrm{ml}$ Depotocin) als signifikant höher als bei den spontan geferkelten Sauen. Die Überlegenheit betrug + 0,41 Absetzferkel je abgesetzten Wurf.

Tabelle 5

Geburtsdauer und Wurfleistungen der Sauen nach unterschiedlicher Geburtsauslösung (Duration of paruturition and litter traits of sows following different induction of farrowing)

\begin{tabular}{|c|c|c|c|c|}
\hline \multirow[t]{2}{*}{ Merkmal } & \multicolumn{3}{|c|}{ Art der Geburtsauslösung } & \multirow[b]{2}{*}{ spontan } \\
\hline & I: $50 \mu \mathrm{g}$ Cloprostenol & $\begin{array}{l}\text { II: } 50 \mu \mathrm{g} \mathrm{Cl}+1 \mathrm{ml} \\
\text { Depotocin }\end{array}$ & $\sum \mathrm{I}+\mathrm{II}$ & \\
\hline Tierzahl (St.) & 369 & 187 & 551 & 170 \\
\hline Geburtsdauer (min.) & $276 \pm 172$ & $216 \pm 141$ & $255 \pm 165$ & $261 \pm 139$ \\
\hline $\begin{array}{l}\text { Geburtsdauer je insg. } \\
\text { geb. Ferkel (min) }\end{array}$ & 24,7 & 22,2 & 23,96 & 24,96 \\
\hline insg. geb. Ferkel/ Wurf (St.) & $11,16 \pm 3,14$ & $9,74 \pm 3,63$ & $10,67 \pm 3,38$ & $10,45 \pm 3,04$ \\
\hline leb.geb. Ferkel /Wurf (St.) & $10,59 \pm 2,92$ & $9,44 \pm 3,51$ & $10,19 \pm 3,18$ & $10,06 \pm 3,16$ \\
\hline tot geb. Ferkel /Wurf (\%) & 5,1 & 3,1 & 4,5 & 3,7 \\
\hline abges. Ferkel /Wurf (St.) & $8,83 \pm 1,95$ & $8,54 \pm 3,13$ & $8,73 \pm 2,42^{2}$ & $8,32 \pm 2,78^{b}$ \\
\hline
\end{tabular}

In Tabelle 6 werden die analog zum ersten Versuchsabschnitt registrierten Ergebnisse zur Tiergesundheit ausgewiesen.

Tabelle 6

Tiergesundheitliche Parameter nach unterschiedlicher Geburtsauslösung ( Parameters of animal health following different induction of parturition)

\begin{tabular}{|c|c|c|c|c|}
\hline \multirow[t]{2}{*}{ Merkmal } & \multicolumn{3}{|c|}{ Art der Geburtsauslösung } & \multirow[t]{2}{*}{ spontan } \\
\hline & I: $50 \mu \mathrm{g}$ Cloprostenol & II: $50 \mu \mathrm{g} \mathrm{Cl}:+1 \mathrm{ml}$ Depotocin & $\mathrm{I}+\mathrm{II}$ & \\
\hline \multicolumn{5}{|l|}{ Anteile von Sauen (\%) } \\
\hline mit Milchmangel & 9,3 & 10,6 & 9,5 & 10,0 \\
\hline norm. Körperinnentemp. & 57,0 & 54,5 & 56,0 & 54,4 \\
\hline leicht erhöhte Temperatur & 24,1 & 26,3 & 24,9 & 31,1 \\
\hline stark erhöhte Temperatur & 18,9 & 19,2 & 19,1 & 14,4 \\
\hline ohne Ausfluß & 51,1 & 58,8 & $56,0^{2}$ & $47,8^{b}$ \\
\hline mit leichtem Ausfluß & 38,2 & 35,3 & 37,1 & 35,6 \\
\hline mit schwerem Ausfluß & 7,7 & 5,9 & $6,99^{\circ}$ & $16,7^{\circ}$ \\
\hline \multicolumn{5}{|l|}{ Anteile von Würfen (\%) } \\
\hline ohne notwend.Ferkelbehandlung 2 & 25,5 & 29,0 & $26,8^{2}$ & $37,7^{\circ}$ \\
\hline Behandlung einzelner Ferkel & 61,4 & 51,6 & 57,7 & 44,2 \\
\hline Behandlung der Mehrzahl Ferkel & 13,1 & 19,4 & 15,5 & 22,1 \\
\hline
\end{tabular}

Zwischen den mit Cloprostenol allein bzw. kombiniert behandelten Sauen ergaben sich bei den ausgewerteten Kriterien keine bedeutenden Unterschiede. Gegenüber den Spontangeburten erwies sich die biotechnische Geburtsauslösung in mehreren tiergesundheitlichen Parametern als einflußreich. Die Versuchsgruppen wiesen einen deutlich höheren Anteil von Tieren auf, die frei von Ausfluß waren; der Anteil schwerer Ausflüsse war erniedrigt.

Die Würfe nach biotechnischer Auslösung erbrachten bezüglich der Behandlung erkrankter Ferkel eine differenzierte Häufigkeitsverteilung auf die gebildeten Klassen zu solchen aus spontanen Geburten. Die Anteile von Würfen ohne notwendige Therapie- 
maßnahmen sowie derjenigen mit der Mehrzahl behandelter Ferkel waren zwar erniedrigt; dafür stieg der Anteil von Würfen, in denen einzelne Ferkel erkrankungsbedingt behandelt werden mußten. Diese Tendenzen waren insbesondere bei den mit Clopostrenol allein ausgelösten Würfen erkennbar.

\section{Diskussion}

In der Sauenhaltung erbringen Bewirtschaftsprogramme, die auf dem periodenweisen Abferkelsystem basieren, eine Reihe von tiergesundheitlichen, arbeitswirtschaftlichen und ergebniswirksamen Vorteilen. Diese stehen im Zusammenhang mit der konsequenten Einhaltung des „Alles - rein - alles - raus - „Prinzips bei der Belegung der Abferkel- und Aufzuchtabteile. Feste Hygieneregime sowie standardisierte und damit zertifizierbare Arbeitsabläufe tragen zur Sicherung der Tiergesundheit und einer wirtschaftich effizienten Ferkelerzeugung bei. Im vorliegenden Zusammenhang erwies sich die partielle Geburtsinduktion mittels erprobter Biotechnika als vorteilhaftes Instrumentarium zur Gleichschaltung der Geburtseintritte innerhalb der abferkelnden Tiergruppen. Mittels einer einmaligen Injektion des $\mathrm{PGF}_{2 \alpha}-$ Analogons Cloprostenol nahe dem physiologischen Termin, d.h. vorzugsweise ab 114. Tr.T., konnten unerwünscht lange Tragezeiten über 116 Tage recht sicher ausgeschlossen werden.

Übereinstimmend zu früheren Feldstudien und vorliegenden Praxiserfahrungen konzentrierten sich die ab 114. Tr.T. ausgelösten Geburten auf die Zeitspanne bis 36 Stunden nach Cloprostenol; die Partusrate 36 lag über 94 \% (ROOST u.a., 1986; SCHNURRBUSCH und HÜHN, 1994). Gegenüber der bislang als Standard eingesetzten Cloprostenol - Dosis von $175 \mu \mathrm{g}$ je Partussau erbrachte die Reduzierung auf $100 \mu \mathrm{g}$ und weiterhin auf $50 \mu \mathrm{g}$ die gleiche geburtsauslösende Wirkung. Damit wurden frühere Untersuchungsergebnisse und Orientierungswerte erhärtet, wonach bereits $87,5 \mu \mathrm{g}$ Cloprostenol (POOL u.a., 1980) bzw. $125 \mu \mathrm{g}$ Cloprostenol für eine effektive Geburtsauslösung ausreichen (ELZE und SCHLEWITZ, 1995).

Während der Abferkelperioden vermindert die konsequente Geburtsüberwachung das Risiko von Totgeburten, perinatalen Verlusten sowie lebensschwachen, nicht aufzuchtfähigen Ferkeln (HELLWIG, 1998). Das gleichzeitige Abferkeln der Sauen bot im Untersuchungsbetrieb den Vorteil, überzählige Ferkel umsetzen oder Würfe von leistungsschwachen bzw. erkrankten Sauen auflösen zu können und kleine Würfe aufzustocken. Diese Maßnahmen trugen dazu bei, daß die Sauen nach biotechnischer Geburtsauslösung eine höhere Aufzuchtleistung mit mehr Absetzferkeln je Wurf erreichten als die Stallgefährtinnen nach spontaner Abferkelung. Die Überlegenheit der Wurfgröße beim Absetzen nach fünfwöchiger Säugezeit betrug 0,51 (1. Versuchsdurchgang) bzw. 0,41 Ferkel je abgesetzten Wurf (2. Versuchsdurchgang) $(p<0,05)$. Der Effekt einer erkrankungsärmeren Aufzucht nach gleichgeschalteten Abferkelterminen wurde wiederholt beobachtet (BILKEI, 1993). Zugleich wirkte der Ausschluß3 von verlängerten Tragezeiten durch die Geburtsinduktion vorbeugend auf das Vorkommen von Puerperalerkrankungen. Damit wurden frühere Verfolgsuntersuchungen bestätigt, die bei fortlaufender Anwendung der Geburtensynchronisation 
eine rückläufige Tendenz des Vorkommens des Mastitis - Metritis - AgalaktieKomplexes (MMA-Syndrom) erkennen ließen (HÜHN, 1992).

Mit dem Anstieg der Geburtsdauer erhöhte sich die Totgeburtenrate, worüber bereits MARONDE (1989) nach Untersuchungen unter vergleichbaren Bedingungen berichtete. Verlängerte sich im vorliegenden Ferkelerzeugerbetrieb die Zeit von der Austreibung des ersten bis zum letzten Ferkel über einen Zeitraum von ca. 4 Stunden hinaus, dann verendeten sub partu anstatt ca. 4 bis zu 10 Prozent der ausgetragenen Ferkel. Der von anderen Autoren beschriebene Effekt einer medikamentösen Geburtsbeschleunigung durch das Langzeitxytocin Depotocin (SCHULZ, 1993) blieb vorliegend aus, wenn die Geburtsdauer auf die Anzahl insgesamt geborener Ferkel bezogen wurde. Jedoch erbrachte die zusätzliche Injektion von Depotocin an Sauen, die nicht innerhalb von 24 Stunden nach der PGF $2 \alpha$ - Applikation mit der Geburt begonnen hatten, eine weitere Verkürzung der Abferkelperiode im Vergleich zur alleinigen Verabreichung von Cloprostenol. Bezogen auf den Wirkstoff Carbetocin, erwiesen sich $1 \mathrm{ml}$ Depotocin (enthält 0,07 mg Carbetocin) als wirksame Dosis. Damit wurden die von WÄHNER und HÜHN (1996) zusammengefaßten Anwendungsbeispiele anderer Schweinezuchtbetriebe bestätigt. Frühere Empfehlungen zur Depotocin - Dosierung für die Indikation Geburt/ Puerperium orientierten auf das Doppelte, nämlich 0,175 mg Carbetocin je Partussau (BERNHARD u.a., 1993).

Die vorliegenden Untersuchungsergebnisse lassen für die praktische Nutzung der Geburtensynchronisation die folgenden Schlußfolgerungen $\mathrm{zu}$ :

1. Die biotechnische Lenkung der Geburtseintritte mittels $P F_{2 \alpha}$ allein oder kombiniert mit Depotocin hat vorrangig reproduktionssteuernde Funktionen. Sie unterstützt in vorteilhafter Weise spezielle tierhygienische Konzepte und Managementmaßnahmen zur Sicherung hoher Aufzuchtergebnisse im Abferkelstall, vermag diese jedoch nicht zu ersetzen. Das aus tiergesundheitlichen, arbeitswirtschaftlichen und produktionstechnischen Gründen angezeigte ,Alles - rein - alles - raus „- Verfahren bei der Bewirtschaftung der Abferkel- und Aufzuchtställe läßt sich sinnvoll unterstützen, wenn nicht nur die Geburten, sondern auch die vorausgegangenen Belegungen/Besamungen der Sauen gruppenweise synchronisiert waren.

2. Weitere Voraussetzungen für die erfolgreiche Einbeziehung in das fortpflanzungsbiologische Herdenmanagement bestehen im sachgemäßen, kontrollierten Einsatz der hierfür erprobten und zugelassenen Biotechnika und einer darauf abgestimmten Betriebsorganisation und Ablaufplanung im sauenhaltenden Betrieb. Dazu zählen die intensive Überwachung der Geburten vornehmlich während der Hauptabferkelperioden sowie die Beherrschung und gezielte Durchführung des Wurfausgleiches.

3. Bei den durchgeführten Untersuchungen erwies sich für die Cloprostenol-Injektion ab 114. Trächtigkeitstag die erprobte Niedrig-Dosierung von $50 \mu \mathrm{g}$ als wirksam. Beim kombinierten Behandlungsverfahren, welches den Einsatz von Depotocin in Abstand von ca. 24 Stunden nach PGF $_{2 \alpha}$ vorsieht, sind $0,07 \mathrm{mg}$ Carbetocin, enthal ten in $1 \mathrm{ml}$ Depotocin, völlig ausreichend und als empfehlenswerter Dosierungsvor-
schlag anzusehen. 


\section{Literatur}

BERNHARD, A.; SCHULZ, J.; GUTJAHR, St.; EULENBERGER, K.:

Indikationen für die Anwendung eines Depotoxytozin-Präparates in der tierärztlichen Praxis. Tierärztl. Umschau, Konstanz 48 (1993) 7, 446 - 453

BILKEI, G.:

Der Einfluß der Biotechnik auf die Reproduktion in Schweinezuchtbeständen. Der Praktische Tierarzt, Hannover 74 (1993) 33-37

ELZE, K.; SCHLEWITZ, R.: Maßnahmen der Zuchtsauenbestandsbetreuung zur Vorbeuge von perinatalen Ferkelverlusten. Tierärzt. Umschau, Konstanz 50 (1995), 175 - 178

HEIDLER, W.; HÜHN, U.: Untersuchungen über die Trächtigkeitsdauer beim Schwein und ihre Beziehungen zur Wurfgröße in industriemäßig produzierenden Anlagen. Arch. Tierz., Berlin 22 (1979) 3, 167 - 175

HELLWIG, E.G.: Ferkelverluste - Ursachen und Gegenmaßnahmen. Schweinewelt, Rheinbach 23 (1998) 4, 19 - 22

HOFMANN, F.: Mittel zur Förderung des Gesundheitszustandes im Schweinebestand. Zeitschrift f. Schweinezucht, Neudamm 42 (1935) 41,1 - 3

HOFMANN, F.: Planmäßige Schweineerzeugung mit Hilfe des periodenweisen Abferkelsystems. Wiss. Zeitschr. Univ. Jena, math.-naturwiss. Reihe 9 (1959/60) 261 - 268

HÖRÜGEL, K.; HÜHN, U.; LAASCH, F.-O.: Untersuchungen zur Geburtsinduktion bei Sauen mittels eines kombinierten Behandlungsregimes aus Cloprostenol - Jenapharm und Oxytozin. Mh. Vet.-Med., Jena 43 (1988) 264 - 266

HOLTZ, W. :

Neue Erkenntnisse zur Beeinflussung der Geburt beim Schwein. Tag.-Ber. Akad. Landwirtsch. - Wiss., HƯHN, U.: Berlin (1988) 273, $141-152$

Ergebnisse mehrjähriger Verfolgungsuntersuchungen zur Geburtensynchronisation in Sauenherden. Mh. Vet.-Med., Jena 47 (1992) 317 - 323

HÜHN, R.; ELZE, K.; ETZRODT, F.; FRANZ, J.; HÜHN, U.: Untersuchungsergebnisse zur biotechnischen Geburtsauslösung beim Schwein mittels eines synthetischen Prostaglandin - $F_{2 \alpha}$ Analogans. Arch. Tierz., Berlin 24 (1981) 1, 15 - 23

HÜHN, U.; KÖNIG, I.: Stand der Praxisanwendung und Weiterentwicklung der Geburtensynchronisation zur Intensivierung der Absetzferkelproduktion. Tierzucht, Berlin 43 (1989) 5, 215 - 217

LUSKY, K.; HÜHN, U.; MAASS, P.; GERICKE, R.; NOACK, F.R.: Untersuchungen zur klinischen Erprobung von Cloprostenol des VEB Jenapharm Partussychronisation beim Schwein. Mh. Vet.-Med., Jena 42 (1987) 278 - 280

MARONDE, J.:

Untersuchungen zur betrieblichen Optimierung der Fortpflanzungstechnologie in einer Stammzuchtanlage der Schwerfurter Fleischrasse durch Nutzung der Rechentechnik. Univ. Rostock, Diss., 1989

PODANY, J.; MAJERCIAK, P.; STEJSKAL, J.; STEFAN, M.:

Fertility of sows with cloprostenol - induced parturition in all - in - all - out management system. Pig News and Information, Wallingford 8 (1987) 23 - 27

POOL, SH.; COPELAND, D.D.; GODKE, R.A.: Induced farrowing in commercial gilts with three dose levels of cloprostenol (ICI - 80 996). J. Anim. Sci., Albany, N.Y. 49 (1980) Suppl. 1, 327

RITTER, E.:

Dr. Fritz HOFMANN'S bahnbrechendes Wirken in der Mark- die Entwicklung der Schweineleistungskontrolle. Festschrift „85 Jahre organisierte Schweinezucht in Brandenburg 1913 1998“, Schönow und LVAT Ruhlsdorf / Gr. Kreutz (1998), 33 - 37 
ROOST, H.; WEICHELT, UTE; WEICHELT, K.; HETT, H.; HÜHN, U. ; MAXIMOWITZ, E.:

Erfahruungen und Ergebnisse bei der Durchführung der partiellen Geburtsinduktion bei Sauen der SCHEWE, H.: Linie F 150 in einer 1000 er Sauenanlage. Mh. Vet.-Med., Jena 41 (1986) 118 - 121

Integration der 1275 er Sauenzuchtanlagen in das Zuchtprogramm des Hybridschweinezuchtverbandes Nord/Ost e.V. Tag.-Ber. „25 Jahre erfolgreiche Schweinezucht in 1275er Sauenanlagen." Uns Swintiding, Neubrandenburg 5 (1998) Sonderheft, 3 - 11 SCHLEGEL, W:

Biologische Aspekte für die Reproduktion des Sauenbestandes. In: PFEIFFER, H. (Federführung): Schweinezucht, Deutscher Landwirtschaftsverlag Berlin, 1. Aufl. (1978) 197 - 206

SCHNURRBUSCH, U.; HÜHN, U.:

Fortpflanzungssteuerung beim weiblichen Schwein. Gustav Fischer Verlag Jena, Stuttgart (1994) 123 131

SCHULZ, J.

Medikamentöse Maßnahmen in der Geburtshilfe. In: Geburtshilfe bei Haustieren, Hrsg. Von W. WÄHNER, M.; HÜHN, U.:

Geburtensteuerung bei Partussauen mit Prostaglandinen und Depotocin. Tag.-Ber. 2. Bernburger Biotechnik-Workshop, FH Anhalt (1996), 51 - 58

Eingegangen: 16.02 .1999

Akzeptiert: 01.06.1999

Anschriften der Verfasser

Prof. Dr. UWE HÜHN

Gustav-Frölich-Allee 29

D-18196 Dummerstorf

Tzl. Dr. GOTTFRIED GEY

Niklotstraße 32

D-18273 Güstrow 


\title{
Buchbesprechung
}

\author{
Lehrbuch der Schweinekrankheiten
}

HANS PLONAIT und KLAUS BICKHARDT (Hrsg.)

2. neubearbeitete Auflage, 602 Seiten, 318 Abbildungen davon 57 farbig, 63 Tabellen, Parey Buchverlag Berlin im Blackwell Wissenschaftsverl. GmbH, Berlin - Wien, 1997, ISBN 3-8263-3149-4, 168,00 DM, 1226,00 ÖS, $155,00 \mathrm{SFr}$

Trotz prophylaxeorientierter Schweineproduktion und derzeit zunehmenden, einschneidenden Regelungen zur Haltung, Medikamentenanwendung, der Schachtverwertung und des Tierschutzes, sieht sich der Tierarzt und der Schweinehalter mit Therapienotwendigkeiten konfrontiert. Es ist das Verdienst der namhaften Herausgeber und Koautoren mit dem „Lehrbuch der Schweinekrankheiten“ nunmehr in 2. völlig überarbeiteter Fassung, einen umfassenden Überblick der wichtigsten Erkrankungen des Schweines vorzulegen, der weit über das Anliegen eines Lehrbuches hinausgeht. Das kranke Schwein, in seinem Bestand und sein Krankheitsbild, steht im Mittelpunkt. Von ihm ausgehend sind diagnostische, therapeutische, und prophylaktische Entscheidungen zu treffen. Diesem Buchansatz liegt zugrunde, daß Krankheiten an typischen Erscheinungen erkennbar und Tierbestände biologische Einheiten sind. Da sich dieses Buch neben Auszubildenden bewußt an einen breiteren Leserkreis wendet, haben sich die Autoren um eine verständliche, praxisrelevante Darstellungsweise bei der Beschreibung der einzelnen Krankheitsbilder bemüht, um die zugrunde liegenden Vorgänge verständlich zu machen und Anlcitungen zum praktischen Handeln zu geben. Dieses Buch setzt zwar tierärztliches Grundwissen voraus, bietet jedoch auch den Schweinehaltern wertvolle Informationen, ohne bei ihnen den Tierarzt ersetzen zu wollen. Die Überarbeitungen beziehen sich vor allem auf neuartige Krankheiten und die Weiterentwicklung der präventiven Veterinärmedizin. Die Anzahl der Abbildungen wurde verdoppelt und jüngere Literatur, vor allem aus dem englischsprachigen Raum, wurde ergänzt.

Im ersten, allgemeinen Hauptteil werden in vier Kapiteln die Aufgaben des Tierarztes im Schweinebestand, der Einfluß der Haltungsbedingungen auf das Krankheitsgeschehen, der Umgang des Tierarztes mit Schweinen und die unterschiedlichen therapeutischen Techniken beschrieben. Der spezielle Teil enthält mit zahlreichen Unterabschnitten die Darstellung folgender Krankheitsgruppen: Hautkrankheiten und Hautveränderungen, fieberhafte Allgemeinerkrankungen, bakterielle Infektionen, Erkrankungen des Atmungsapparates, des Herz-Kreislaufsystems, Blutkrankheiten, Pathophysiologie und Diagnostik, Erkrankungen und Störungen des Zentralnervensystems, bakteriell bedingte ZNS-Erkrankungen, Muskelerkrankungen, Gliedmaßen- und Skeletterkrankungen, Erkrankungen der Verdauungsorgane und des Abdomens, der Harnorgane, Fortpflanzungsphysiologie und Gynäkologie der Sau, Geburt, Puerperium und perinatale Verluste, Erkrankungen des Gesäuges, der Geschlechtsorgane und Operationen an den Fortpflanzungsorganen des Ebers. Im abschließenden Kapitel werden Fragen des Infektionsschutzes, der Sanierung und der planmäBigen Bestandsbehandlung erörtert. Eine detaillierte Gliederung, die große Anzahl von teils farbigen Abbildungen und aussagekräftige Tabellen, tragen wesentlich zum Verständnis der Texte bei. Bei den einzelnen Erkrankungen werden Ätiologie, Phatogenese, klinische Erscheinungen und Verläufe, Diagnose, Therapie und Prophylaxe besprochen.

Das sehr praxisrelevante Buch informiert über neuesten Wissenschafts- und Erkenntnisstand auf diesem Gebiet. Es ist sowohl für die Aus- und Weiterbildung als auch für den praktischen Tierarzt und den interessierten Schweinehalter als unentbehrliches Standardwerk sehr zu empfehlen. 